\title{
Constraints on the Release History of Solar Energetic Particles by Flux-tube Variations
}

\author{
N. Agueda ${ }^{1}$ and K.-L. Klein ${ }^{2}$ \\ ${ }^{1}$ DAM/ICC, Universitat de Barcelona, Spain \\ ${ }^{2}$ Observatoire de Paris, LESIA, CNRS-UMR, France
}

\begin{abstract}
We present the analysis of a large solar near-relativistic ( $>50 \mathrm{keV})$ electron event observed by the Wind spacecraft on 1998 April 20. In-situ data show variations of the local magnetic field direction accompanied by changes in the topology of the observed electron pitchangle distributions. These suggest changes in the magnetic flux tubes scanned by Wind. Using simulations of the interplanetary particle transport, we model the early rising phase of the electron event, from 10:30 to 11:00 UT, and infer the propagation conditions and the injection history of the first arriving $50-82 \mathrm{keV}$ solar electrons. The results reveal a prompt ( $\leqslant 1 \mathrm{~min})$ release in coincidence with the soft X-ray and type III radio bursts, suggesting that the first arriving electrons were flare-accelerated.
\end{abstract}

Keywords. Sun: particle emission, interplanetary medium, Sun: flares

\section{Introduction}

The solar energetic particle (SEP) event on 1998 April 20 was observed during quiescent interplanetary (IP) conditions: no IP coronal mass ejections (CMEs) passed Earth during the previous $\sim 10$ days (Cane \& Richardson 2003) and the in-situ particle intensities were near solar-quiet levels for roughly a week before the event (Tylka et al. 1999).

The SEP event was presaged by a prominence eruption at the southwest limb of the Sun, where an M1.4 X-ray flare was reported at 10:21 UT. Distinct radio emission episodes included a moving type IV burst (9:40-10:00 UT), followed by a series of decametric-hectometric (DH) type III bursts (10:03-10:33 UT), and a type II radio burst at 5-10 MHz after 10:25 UT (Maia et al. 2000; Klassen et al. 2002). The DH type III bursts were weak at frequencies above $2 \mathrm{MHz}$, suggesting that the parent active region was occulted behind the solar limb. A partial halo fast $(1863 \mathrm{~km} / \mathrm{s})$ CME was seen above 3 solar radii at 10:04 UT (Vourlidas et al. 1999). The expanding CME loops were imaged directly at radio wavelengths, and the emission ascribed to non-thermal synchrotron emission from $\sim 0.5-5 \mathrm{MeV}$ electrons in the CME magnetic field (Bastian et al. 2001).

\section{In-situ Observations}

Fig. 1 (left) shows the 50-82 keV electron omni-directional intensities observed by Wind/3DP on 1998 April 20, and the pitch-angle distributions (PADs). The following panels show the measured solar wind speed and the IP magnetic field strength and direction in the GSE coordinate system. It can be seen that during the rising phase of the event the magnetic field direction changed significantly. We identified four periods of time in Fig. 1, with the following $\vec{B}$ mean latitude and longitude: $\left(-35^{\circ}, 242^{\circ}\right) ;\left(-66^{\circ}, 80^{\circ}\right)$, $\left(-30^{\circ}, 245^{\circ}\right)$, and $\left(32^{\circ}, 230^{\circ}\right)$. These four periods showed different PADs topologies, that is, antisunward PADs with a positive polarity, bidirectional PADs, antisunward PADs with a negative polarity and antisunward PADs with a positive polarity, respectively. 

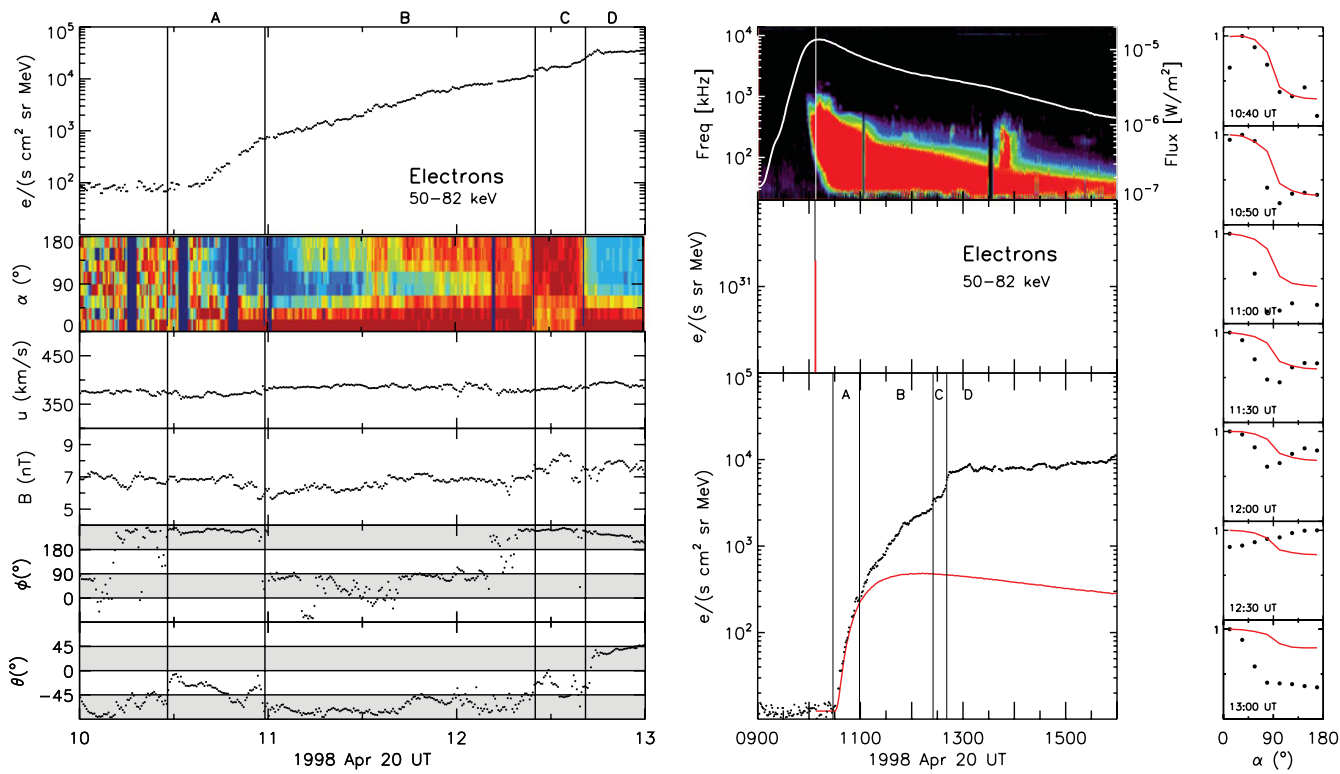

Figure 1. Left: Electron 50-82 keV omni-directional intensities and PADs; solar wind speed; magnetic field strength and direction (longitude and latitude) in the GSE coordinate system. Center: $20 \mathrm{kHz}-14 \mathrm{MHz}$ Radio spectrum and soft X-ray flux profile (white curve) expressed in terms of solar release time $\left(t_{\mathrm{SRT}}=t_{\mathrm{AU}}-500 \mathrm{~s}\right)$; Best fit injection function; Omni-directional intensities (black: observations, red: fit). Right: Observed and modeled normalized PADs.

\section{Results}

We used an inversion method (Agueda et al. 2008) to fit the PADs observed by Wind from 10:30 to 11:00 UT. The method utilizes Green's functions of IP transport (Agueda et al. 2012) to infer the best fit injection function and the value of the electron mean free path in the IP medium. The method reveals a prompt $(\leqslant 1 \mathrm{~min})$ release of electrons (indicated by the red vertical line in Fig. 1, central panel) at the time of the soft X-ray and type III radio bursts. The inferred value of the radial mean free path is $0.05 \mathrm{AU}$. Beyond 11:00 UT, the modeled intensities are unable to explain the observations, since the event is dominated by changes in the magnetic flux tube at least until 12:41 UT. We conclude that the duration and timing of the injection suggest the first arriving electrons were flare-accelerated, and got access to the field line magnetically connecting Wind to the Sun along diverging open field lines in the parent active region.

\section{References}

Agueda, N., Vainio, R., Lario, D., \& Sanahuja, B. 2008, ApJ, 675, 1601

Agueda, N., Vainio, R., \& Sanahuja, B. 2012, ApJ (Supplement), 202, id: 18

Bastian, T. S., Pick, M., Kerdraon, A., \& Maia, D., Vourlidas A. 2001, ApJ (Letters), 558, L65

Cane, H. V. \& Richardson, I. G. 2003, J. Geophys. Res., 108, 1156

Klassen, A., Bothmer, V., Mann, G., Reiner, M. J., Krucker, S., Vourlidas, A., \& Kunow, H. 2002, A\&A 385,1078

Maia, D., Pick, M., Vourlidas, A., \& Howard, R. 2000, ApJ, 528, L49

Tylka, A. J., Reames, D. V., \& Ng, C. K. 1999, Geophys. Res. Lett., 26, 2141

Vourlidas, A., Maia, D., Pick, M., \& Howard, R. A. 1999, in Magnetic Fields and Solar Processes (ESA SP-448), ed. A. Wilson (Noordwijk: ESA), 1003 